Ann. Zootech., 1974, 23 (4), 503-518.

\title{
EFFETS SÉPARÉS ET CUMULÉS DU NOMBRE DE REPAS ET DE LA TEMPERATURE DU LAIT SUR LES PERFORMANCES DES GHEVREAUX DE BOUCHERIE
}

\author{
P.-M. FEHR et D. SAUVANT \\ avec la collaboration technique de J. Hervieu et A. Cuccr \\ Laboratoire de Recherches de la Chaire de Zootechnie, \\ Institut national agronomique, Paris-Grignon, \\ 16, rue Claude-Bernard, \\ 75231 Paris Cedex 05
}

\section{RÉSUMÉ}

32 chevreaux mâles de race Alpine sont répartis en 4 lots qui reçoivent à partir du $9^{\mathrm{e}}$ jour, exclusivement du lait de remplacement selon les modalités suivantes :
Lot $2 \mathrm{C}: 2$ repas par jour, lait distribué à une to de $35^{-40^{\circ} \mathrm{C}}$
Lot I $\mathrm{C}:$ I
Lot $2 \mathrm{~F}: 2 \quad \ldots \quad-\quad-\quad 6-88^{\circ} \mathrm{C}$.
Lot I F : I

Le lait de remplacement des lots $2 \mathrm{C}$ et $2 \mathrm{~F}$ contient 16 p. roo d'aliment d'allaitement, celui des lots $\mathrm{x}$ C et $\mathrm{x}$ F, 24 p. Ioo. Les animaux ingérant du lait froid ont la possibilité de se placer sous une lampe infra-rouge pendant les repas.

Les chevreaux sont abattus à un poids compris entre 9 et $10,5 \mathrm{~kg}$ et à un âge de 28 à 37 jours.

La distribution quotidienne d'un repas au lieu de deux et la consommation de lait froid diminuent légèrement la consommation alimentaire journalière, la vitesse de croissance, et l'état d'engraissement des carcasses. L'ingestion de lait froid améliore l'efficacité de l'utilisation alimentaire. L'effet du nombre de repas et de la température du lait s'ajoutent dans la plupart des cas.

La distribution d'un seul repas par jour ou de lait froid semble être des techniques intéressantes à utiliser dans la production du chevreau de boucherie en raison de l'économie de maind'œuvre qu'elles permettent tout en maintenant un niveau de performances satisfaisant.

Par ailleurs, l'interprétation des résultats par l'analyse de données en composantes principales a permis de mieux préciser les caractéristiques de la croissance des chevreaux mâles au cours du premier mois. 


\section{INTRODUCTION}

Dans les élevages caprins français qui sont essentiellement orientés vers la production laitière, les chevreaux n'étant pas destinés à la reproduction sont engraissés et abattus généralement à un poids compris entre 6 et I I $\mathrm{kg}$ et à un âge variant de I5 à 35 jours. Cette production, bien qu'elle puisse représenter de I 5 à 25 p. IoO du produit brut d'un élevage de chèvres (DISSET, I97I) est souvent délaissée en raison de son exigence en main-d'œuvre à une époque où les mises bas et le début de la lactation imposent une surcharge de travail à l'exploitation.

La mise au point de méthodes d'alimentation peu exigeantes en main-d'œuvre paraît donc souhaitable. Or, la distribution d'un repas par jour de lait entier ou de lait de remplacement à des veaux (ACKERMAN et al., I969; BETTENAX, I97 I BURT, I968; EEckHou'T et Buysse, I969 et I97I ; KHOURI, I969; LEAVER et Yarrow, I972 $a$ et $b$; OWEN, Plum et Harris, I965; OWENS et Stake, I97I ; White et RadCLIFFE, I970 ; WILLETT et al., I969; WII,SON, I968; WoOD et al., I97I ; HodEN et JoURNET, I972) permet dans la plupart des cas d'obtenir des performances satisfaisantes de croissance, d'efficacité alimentaire et de qualité de carcasses tout en réduisant la main-d'œuvre. Il en est de même de la consommation de lait de remplacement à température ambiante ou même à plus basse température chez des veaux ou des agneaux (Brisson et Bouchard, I970 ; FLIPOT et al., I972 ; GibB et PENNING, I972 ; LARge et Penning, I967; PenNing et al., I97I et I972; TAYLER et LondSale, Ig69; Theriez et Molenat, I969). De ce fait, nous avons recherché si ces deux méthodes d'élevage peuvent s'appliquer à la production de chevreau de boucherie et si leurs effets petvent ou non s'ajouter. Des résultats partiels de cette expérience ont déjà été rapportés dans une première note (FEHR, I97I).

\section{MATÉRIEL ET MÉTHODES}

L'expérience est réalisée suivant un plan expérimental factoriel de $2 \times 2$ combinant les effets des apports de lait chaud ou de lait froid et de la distribution d'un ou deux repas par jour. 32 chevreaux mâles de race $A l p i n e$ dont les naissances se sont étalées sur 2 semaines sont séparés de leur mère immédiatement après la mise bas. Ils sont répartis en 4 lots de 8 chevreaux, équilibrés suivant le poids à la naissance et la taille de la portée. Les animaux de chaque lot sont alors placés sur paille dans des cases de $\mathrm{I}, 50 \times \mathrm{I}, 50 \mathrm{~m}$ permettant une distribution individuelle d'aliment lacté.

Comme l'indique le tableau $I$, tous les chevreaux subissent le même traitement pendant la première semaine : colostrum de la mère, lait de chèvre et substitution du lait de chèvre par du lait de remplacement.

Dès le $9^{\mathrm{e}}$ jour, les 4 lots reçoivent un régime diffétent :

Lot $2 \mathrm{C}: 2$ repas, lait Chaud

Lot I C : I repas, lait Chaud

Lot $2 \mathrm{~F}: 2$ repas, lait Froid

Lot I $F$ : I repas, lait Froid

Les repas sont distribués à 8 heures et à $\mathrm{I} 7$ heures. Le lait est distribué dans une coupelle qui reste à la đisposition de l'animal pendant $\mathrm{I} / 2$ heure. Les chevreaux à qui un seul repas est distribué consomment un lait de remplacement $50 \mathrm{p}$. roo plus concentré que les autres afin de leur permettre d'ingérer plus de matière sèche par repas. Ils ne reçoivent que le repas du matin. De l'eau est continuellement à la disposition de ces chevreaux. 
Les animaux des lots $2 \mathrm{~F}$ et $\mathrm{I} \mathrm{F}$ qui ingèrent du lait froid ont la possibilité de se placer sous une lampe infra-rouge de $250 \mathrm{~W}$. qui reste allumée au moment du repas et pendant la demi-heure suivante.

Le lait de remplacement distribué chaud est préparé à chaque repas avec de l'eau à une température d'environ $50^{\circ} \mathrm{C}$. alors que le lait de remplacement froid est préparé seulement deux fois par semaine avec de l'eau froide $\left(6\right.$ à $10^{\circ} \mathrm{C}$. $)$. Ce lait est conservé à $4^{\circ} \mathrm{C}$. et distribué à la sortie du réfrigérateur après une rapide homogénéisation.

TABLEAU I

Plan d'alimentation

\begin{tabular}{|c|c|c|c|c|}
\hline & Lot $2 \mathrm{C}$ & Lot $1 \mathrm{C}$ & Lot 21 & Lot $1 \mathrm{~F}$ \\
\hline $\begin{array}{l}\text { jer et } 2 \mathrm{ej} \\
3^{\mathrm{e}} \text { et }{ }^{\circ} \mathrm{j} \\
5^{\circ} \text { et } 7^{\circ} \mathrm{j}\end{array}$ & \multicolumn{3}{|c|}{$\begin{array}{l}\text { Colostrum à volonté } \\
\text { Jait de chèvre à volonté } \\
\text { Transition }\left\{\begin{array}{l}\text { lait de chèvre } \\
\text { lait de remplacement }\end{array}\right.\end{array}$} & $\begin{array}{l}-10^{\circ} \mathrm{C} \\
-10^{\circ} \mathrm{C}\end{array}$ \\
\hline $\mathrm{S}^{\mathrm{e}} \mathrm{j}$ & $\begin{array}{c}\text { I.ait } \\
\text { de remplacement } \\
\text { à } 12 \text { p. } 100 \\
\text { à volonté } \\
\text { 'repas } \\
35^{\circ}-10^{\circ} \mathrm{C}\end{array}$ & $\begin{array}{c}\text { Lait } \\
\text { de remplacement } \\
\text { à } 16 \text { p. } 100 \\
\text { à volonté } \\
2 \text { repas } \\
35^{\circ}-40^{\circ} \mathrm{C}\end{array}$ & $\begin{array}{l}\text { Lait } \\
\text { de remplacement } \\
\text { à } 12 \mathrm{p} .100 \\
\text { à volonté } \\
2 \text { repas } \\
35^{\circ}-40^{\circ} \mathrm{C}\end{array}$ & $\begin{array}{l}\text { Lait } \\
\text { de remplacement } \\
\text { à } 16^{\circ} \mathrm{p} .100 \\
\text { a volonte } \\
2 \text { repas } \\
35^{\circ}-100^{\circ} \mathrm{C}\end{array}$ \\
\hline $9 \mathrm{c}$ & $\begin{array}{l}\text { I ait } \\
\text { de remplacement } \\
\text { à } 16^{2} \text { p. } 100 \\
\text { a volonté } \\
2 \text { repas } \\
35^{\circ}-40^{\circ} \mathrm{C}\end{array}$ & $\begin{array}{c}\text { Lait } \\
\text { de remplacement } \\
\text { à } 24 \text { p. } 1(10) \\
\text { à volonté } \\
1 \text { repas } \\
355^{\circ}+0^{\circ} \mathrm{c}\end{array}$ & $\begin{array}{c}\text { Lait } \\
\text { de remplacement } \\
\text { à } 16 \text { p. } 100 \\
\text { à volonté } \\
2 \text { repas } \\
1 ; 0-80 \%\end{array}$ & $\begin{array}{c}\text { Lait } \\
\text { de remplacement } \\
\text { à } 24 \text { p. } 100 \\
\text { à volonté } \\
1 \text { repas } \\
6^{\circ}-8^{\circ} \mathrm{C}\end{array}$ \\
\hline
\end{tabular}

L'aliment d'allaitement utilisé est un produit du commerce à base de poudre de lait écrémé réengraissé avec du suif, et d'un complément minéral vitaminique. Il contient $26 \mathrm{p}$. roo de matières azotées et 23 p. roo de matières grasses.

La consommation de lait est mesurée individuellement à chaque repas. Les animaux sont pesés deux fois par semaine.

Les chevreaux sont abattus à un poids compris entre $9 \mathrm{~kg}$ et $10,5 \mathrm{~kg}$ et à un âge de 28 à 37 jours après avoir jeûné 24 heures. Le poids de la carcasse avec la tête et des différentes parties du $5^{\mathrm{e}}$ quartier (peau, viscères vidés de leur contenu, en particulier rumen et caillette) est enregistré immédiatement. Des notes appréciant la musculature, l'engraissement et la couleur sont attribuées à chaque carcasse.

L'interprétation des résultats, pour chaque caractère étudié, a été effectuée par analyse de variance en décomposant l'effet du régime en trois effets comme le plan expérimental le permet : effet de la température du lait, effet du nombre de repas et effet de l'interaction entre la température du lait et du nombre de repas. Mais, pour cerner de façon plus objective et plus précise les interrelations entre les résultats de croissance, d'efficacité alimentaire, de qualité de la carcasse et de composition du $5^{\mathrm{e}}$ quartier en liaison avec les facteurs de variation expérimentaux ou non, la méthode d'analyse en composantes principales normées à été utilisée.

Elle permet de résumer fidèlement et de structurer l'information du tableau de données constitué par les différents caractères mesurés sur chacun des 32 chevreaux. Les caractéristiques de cette méthode ont déjà été décrites et son application dans le domaine zootechnique discutée dans une précédente étude (SAUVANT et al., I973). Nous ne rappellerons donc ici que brièvement les principes pratiques de l'interprétation des "sorties graphiques " obtenues par analyse en composantes principales.

Les composantes principales représentent les facteurs de variation essentiels de l'ensemble 
des données expérimentales. Elles sont extraites par ordre décroissant d'importance. En d'autres termes, la première composante explique une plus grande part de la variance des données que la seconde et ainsi de suite. La signification d'une composante est déterminée à l'aide des caractères qui lui sont le plus corrélés et qui, de ce fait, sont graphiquement les plus éloignés de l'origine sur l'axe représentant cette composante. Deux caractères bien expliqués (c'est-à-dire, non-placés à proximité du centre) dans un plan défini par deux axes représentant deux composantes principales. sont corrélées positivement si elles se situent dans des directions voisines à partir du centre et négativement si elles sont dans des directions opposées. Une observation ou un groupe homogène d'observations, en l'occurrence un chevreau ou un lot de chevreaux ont une valeur d'autant plus élevée pour un caractère bien expliqué, que leurs projections sur le plan sont éloignées du centre dans la direction de ce caractère.

\section{RÉSULTATS}

Pendant toute l'expérience l'état sanitaire des animaux est resté très bon. En particulier, aucun cas diarrhéique n'a été enregistré.

\section{Comportement et consommation alimentaire (tab1. 2)}

Les chevreaux qui ne reçoivent qu'un repas par jour s'y habituent très rapidement. En effet, après 2 ou 3 jours ils sont calmes le soir et ne demandent pas à être alimentés. La digestion de leurs ingestats est probablement plus longue en raison des quantités consommées par repas. En effet, à l'abattage, les contenus digestifs dans la caillette sont après 24 heures de jeûne nettement plus importants que ceux observés dans les autres lots.

\section{TABLEAU 2}

Quantités consommées

\begin{tabular}{|c|c|c|c|c|c|c|c|}
\hline \multirow[b]{2}{*}{$\begin{array}{c}\text { Moyenne } \\
\text { par chevreaus }\end{array}$} & \multirow[b]{2}{*}{$\begin{array}{l}\text { Lot } \\
2 \mathrm{C}\end{array}$} & \multirow[b]{2}{*}{$\begin{array}{l}\text { Lot } \\
1 \mathrm{C}\end{array}$} & \multirow[b]{2}{*}{$\begin{array}{l}\text { Lot } \\
2 \mathrm{~F}\end{array}$} & \multirow[b]{2}{*}{$\begin{array}{l}\text { Lot } \\
1 \mathrm{~F}\end{array}$} & \multicolumn{3}{|c|}{ Test de signification } \\
\hline & & & & & $\begin{array}{c}\text { Effet } \\
\text { To du lait }\end{array}$ & $\begin{array}{l}\text { Effet } \\
\text { Nbre } \\
\text { de repas }\end{array}$ & $\begin{array}{c}\text { Effet } \\
\text { Interaction }\end{array}$ \\
\hline 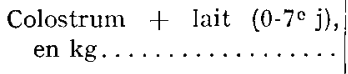 & 6,10 & 5,86 & $6,0^{\prime}$ & 6,28 & NS & NS & is \\
\hline $\begin{array}{l}\text { Aliment d'allaitement } \\
\left(8^{\mathrm{e}} \mathrm{j} \text {, abattage }\right):\end{array}$ & & & & & & & \\
\hline 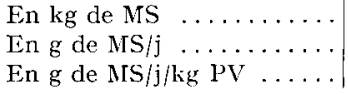 & $\begin{array}{c}6,48 \\
2,7 \\
33,3\end{array}$ & $\begin{array}{c}5,49 \\
234 \\
3 \geq 1\end{array}$ & $\begin{array}{c}5,50 \\
222 \\
31,7\end{array}$ & $\begin{array}{l}5,43 \\
195 \\
26,3\end{array}$ & $\begin{array}{c}+ \\
++++ \\
++++\end{array}$ & $\begin{array}{c}+ \\
++ \\
+++-t\end{array}$ & $\begin{array}{c}+ \\
+\mathrm{is} \\
+++\end{array}$ \\
\hline $\begin{array}{r}\mathrm{Kg} \text { de MS consommés par } \mathrm{kg} \\
\text { de gain }\left(8^{\mathrm{e}} \mathrm{j} \text {, abattage) } .\right.\end{array}$ & 1,22 & 1,23 & $1,1^{4}$ & 1,12 & ++++ & NS & is \\
\hline
\end{tabular}

NS : Non-significatif.

+ : Significatif au seuil de $P \leqslant 0,20$.

++ : Significatif au seuil de $P \leqslant 0,10$.

+++ : Significatif au seuil de $P \leqslant 0,05$.

++++ : Significatif au seuil de $P \leqslant 0,01$. 
L'adaptation des chevreaux à consommer du lait froid est variable suivant les animaux. Un délai de 2 à Io jours est en effet nécessaire pour que les quantités consommées deviennent comparables à celles des chevreaux qui reçoivent du lait chaud. Cette adaptation est plus rapide lorsque le lait est distribué en deux repas. Les chevreaux des lots $2 \mathrm{C}$ et $\mathrm{I} \mathrm{C}$ boivent rapidement leur lait. Par contre, ceux qui reçoivent du lait froid prennent leur repas en 2 ou 3 buvées séparées de courtes périodes passées sous la lampe infra-rouge.

Si la consommation alimentaire au cours de la première semaine n'est guère différente dans les 4 lots, la nature du régime influence significativement les quantités totales d'aliment d'allaitement ingérées du $8 \mathrm{e}$ jour à l'abattage (tabl. 2). Le lot $2 \mathrm{C}$ consomme nettement plus de matière sèche par jour que les autres lots. La distribution d'un repas par jour au lieu de deux repas et l'ingestion de lait froid à la place de lait chaud diminuent significativement la consommation journalière d'aliment d'allaitement rapportée ou non au poids vif.

\section{TABLEAU 3}

Croissance des chevreaux

\begin{tabular}{|c|c|c|c|c|c|c|c|}
\hline \multirow{2}{*}{$\begin{array}{c}\text { Moyenne } \\
\text { par chevreau }\end{array}$} & \multirow{2}{*}{$\begin{array}{l}\text { Lot } \\
2 \mathrm{C}\end{array}$} & \multirow{2}{*}{$\begin{array}{l}\text { Lot } \\
1 \mathrm{C}\end{array}$} & \multirow{2}{*}{$\begin{array}{l}\text { Lot } \\
2 F\end{array}$} & \multirow{2}{*}{$\begin{array}{l}\text { Lot } \\
1 \mathrm{~F}\end{array}$} & \multicolumn{3}{|c|}{ Test de signification } \\
\hline & & & & & $\begin{array}{c}\text { leffet } \\
\mathrm{T}^{\circ} \text { du lait }\end{array}$ & $\begin{array}{c}\text { Effet } \\
\text { Nbre } \\
\text { de repas }\end{array}$ & $\begin{array}{c}\text { Effet } \\
\text { Interaction }\end{array}$ \\
\hline PV à la naissance $(\mathrm{kg})$ & $3,7 / k$ & 3,60 & 3,77 & 3,65 & NS & NS & NS \\
\hline PV au $7^{\mathrm{e}} \mathrm{j}(\mathrm{kg}) \ldots \ldots$ & 4,77 & 4,79 & 4,71 & 4,78 & NS & NS & NS \\
\hline Age à l'abattage $(j)$. & & 30,9 & 32 & 314,5 & NS & NS & +++ \\
\hline PV à l'abattage (kg) & 10,07 & 9,27 & 9,57 & 9,49 & NS & NS & NS \\
\hline Gain de poids $(\mathrm{g} / \mathrm{j})$ & & & & & & & \\
\hline 0 , abattage..... & 177 & 172 & 169 & 155 & $+\lim$ & $\mathrm{NS}$ & $\mathrm{NS}$ \\
\hline $8^{\mathrm{e}} \mathrm{j}$, abattage ...... & 199 & 190 & 189 & 172 & $+\lim$ & $+\lim$ & NS \\
\hline
\end{tabular}

NS : Non-significatif.

+ : Significatif au seuil de $P \leqslant 0,20$.

++ : Significatif au seuil de $P \leqslant 0,10$.

+++ : Significatif au seuil de $P \leqslant 0,01$.

\section{Croissance et efficacité alimentaire (tabl. 3, fig. I)}

Les poids moyens des différents lots sont comparables à la naissance et à 7 jours. Mais les chevreaux qui reçoivent du lait froid subissent un ralentissement de croissance très sensible en $2^{\text {e }}$ semaine, qui persiste plus longtemps dans le lot ne recevant qu'un repas alors que la courbe de croissance de celui qui en reçoit deux tend à rattraper celle des autres lots juste avant l'abattage. Le gain de poids moyen journalier du lot $2 \mathrm{C}$ ingérant deux repas de lait chaud est le plus élevé. En effet, la distribution d'un repas par jour et l'ingestion de lait froid réduisent le gain de poids 
journalier. Toutefois, l'effet de la température du lait est moins marqué sur le gain de poids que sur la consommation alimentaire du 8 e jour à l'abattage : en particulier, le gain de poids journalier du lot $\mathrm{I} F$ est inférieure de $\mathrm{I} 4 \mathrm{p}$. Ioo à celui du lot $2 \mathrm{C}$ alors que la différence correspondante entre les consommations journalières d'aliment d'allaitement est de $20 \mathrm{p}$. Ioo. En conséquence, la distribution de lait froid à la différence du nombre de repas permet de réduire l'indice de consommation exprimé en $\mathrm{kg}$ de matière sèche d'aliment d'allaitement nécessaire à un gain de poids vif d'un $\mathrm{kg}$, donc, d'améliorer l'efficacité de l'utilisation alimentaire.

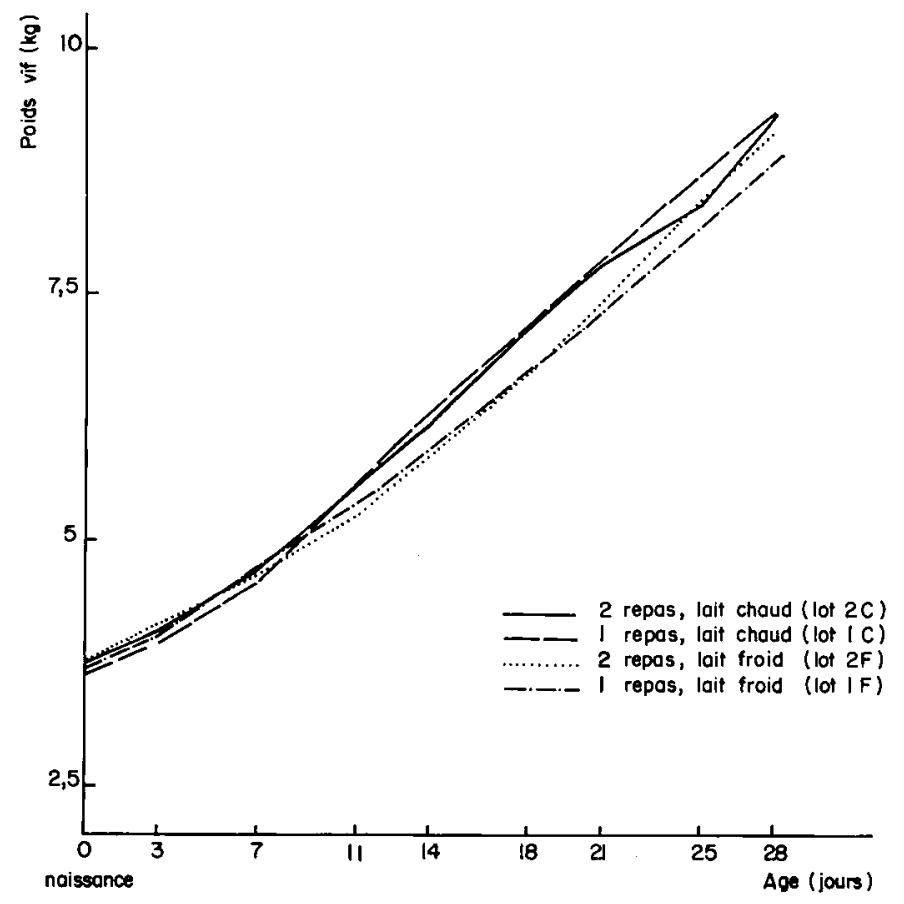

Fig. I. - Courbes de croissance

\section{Qualité de la carcasse et composition du $5^{\mathrm{e}}$ quartier (tabl. 4)}

Comme le poids vif à l'abattage, le poids des carcasses du lot $2 \mathrm{C}$ est supérieur à celui des autres lots ; ce résultat s'explique par la meilleure croissance de ce lot et, en partie aussi, par un âge moyen à l'abattage assez élevé.

Le rendement vrai $\left[\frac{\text { Poids carcasse }}{\text { Poids vif }- \text { bol alimentaire }}\right]$ des carcasses du lot $2 \mathrm{C}$ est aussi supérieur à celui des autres lots probablement en raison d'un développement musculaire et d'un engraissement plus poussés, comme le laissent supposer leur appréciation subjective et le poids de la toilette (gras péritonéal) de ce lot. La distribution de lait froid et d'un seul repas par jour a des effets dépressifs sur l'engraissement de 1a carcasse. Seul le nombre de repas influence significativement l'estimation de la musculature de la carcasse. Par ailleurs, la couleur de la viande ne semble pas être modifiée par la nature du régime. 
Le poids de la caillette vide exprimé en p. Ioo du poids vif n'est pas significativement influencé par l'effet du régime. En revanche, le poids du rumen vide des chevreaux ingérant 2 repas de lait froid est significativement supérieur à celui des autres lots.

TABLEAU 4

Qualité des carcasses des chevreaux

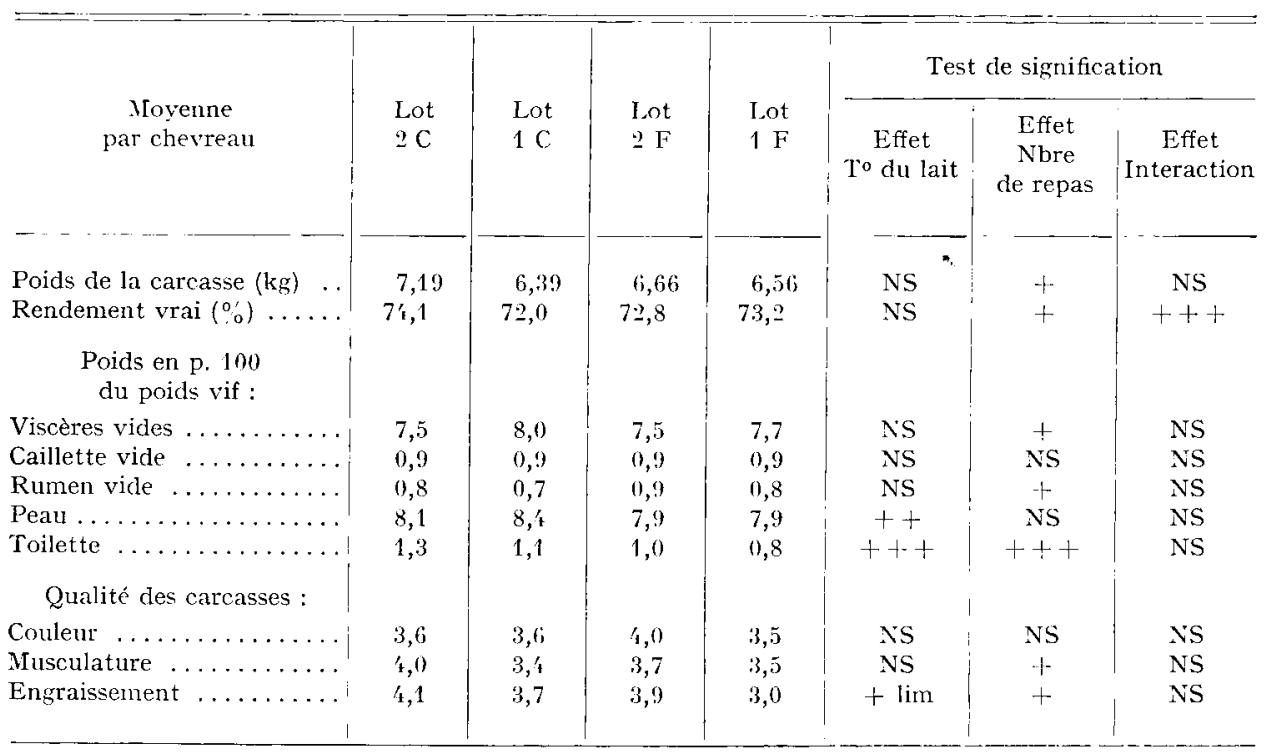

NS : Non-significatif.

+ : Significatif au seuil de $P \leqslant 0,20$.

++- : Significatif au seuil de $P \leqslant 0,10$.

+++ : Significatif au seuil de $P \leqslant 0,01$.

\section{Analyse en composantes principales}

Cette méthode a permis d'analyser très finement la matrice des corrélations (tabl. 5) entre les différents caractères. Puisque certaines corrélations confirment des résultats déjà évoqués précédemment, nous commenterons dans ce qui suit, seulement celles présentant une plus grande originalité à la lumière des sorties graphiques de l'analyse en composantes principales.

Le tableau 6 indique que les 4 premières composantes expliquent près de 75 p. Ioo de la variance totale des données. Sur le même tableau sont indiqués, la moyenne et le coefficient de variation de tous les caractères étudiés ainsi que leurs corrélations avec les composantes principales. Les caractéristiques de la composition corporelle et les notes d'appréciation de la qualité de la carcasse sont moins bien expliquées_par les 4 premières composantes que les autres caractères. Leurs variations 


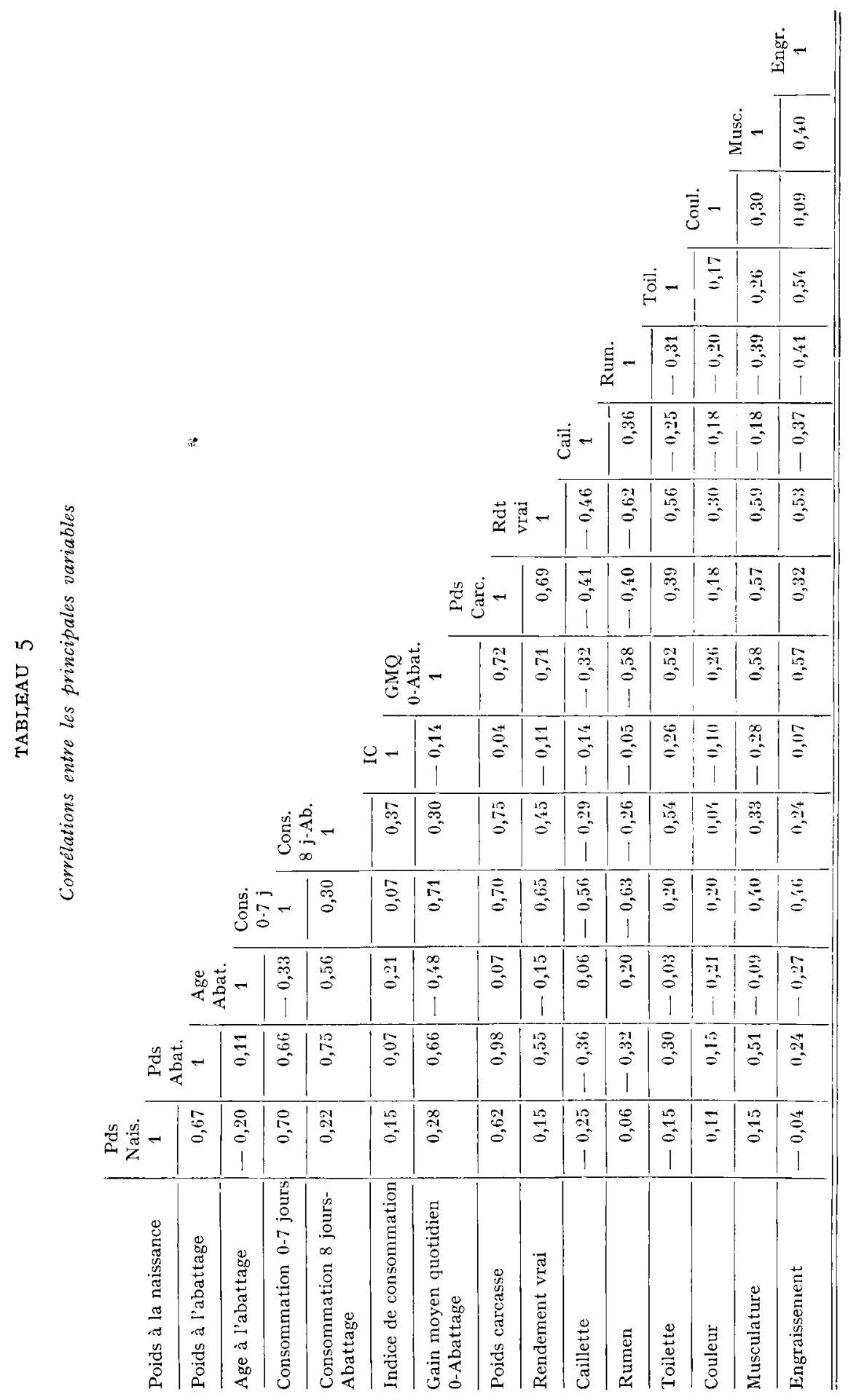




\section{TABLEAU 6}

Coryélations entre les caractères étudiés et les composantes principales

\begin{tabular}{|c|c|c|c|c|c|c|c|}
\hline \multirow[b]{2}{*}{ Caractères } & \multicolumn{7}{|c|}{$N^{\circ}$ de composante } \\
\hline & 1 & 2 & 3 & 4 & 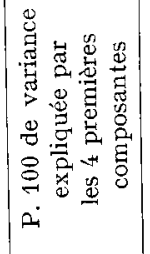 & 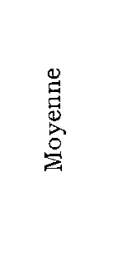 & 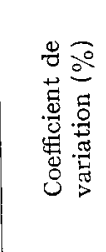 \\
\hline Poids à la naissance ..... & 0,416 & $-0,069$ & 0,785 & 0,137 & 81,3 & 3,69 & 16,8 \\
\hline Poids vif à 7 jours .... & 0,499 & $\longrightarrow 0,179$ & 0,638 & $-0,159$ & 71,3 & 4,63 & 15,2 \\
\hline Poids vif à l'abattage ... & 0,864 & 0,269 & 0,343 & $-0,043$ & 93,8 & 9,6 & 11,9 \\
\hline Age à l'abattage ........ & $-0,084$ & 0,873 & $-0,020$ & $-0,211$ & 81,4 & 32,8 & 12,0 \\
\hline $\begin{array}{l}\text { Gain de poids net à l'abat- } \\
\text { tage ................... } \\
\text { Gain moyen quotidien de }\end{array}$ & 0,886 & 0,318 & $-0,191$ & $-0,127$ & 93,9 & 5,48 & 16,2 \\
\hline $\begin{array}{l}\text { Gain moyen quotidien de } \\
0 \text { à l'abattage } \ldots . . . \ldots \text {. }\end{array}$ & 0,839 & $-0,281$ & $-0,135$ & 0,046 & 80,3 & 168,31 & 16,6 \\
\hline $\begin{array}{l}\text { Gain moyen quotidien de } \\
8 \text { jours à l'abattage } \ldots \\
\text { Consommation de lait de } 0\end{array}$ & 0,732 & 0,574 & - 0,038 & $-0,294$ & 95,3 & 4,84 & 16,1 \\
\hline $\begin{array}{l}\text { Consommation de lait de } 0 \\
\text { à } 7 \text { jours } \ldots \ldots \ldots \ldots \ldots\end{array}$ & 0,789 & $-0,323$ & 0,183 & 0,216 & 80,7 & 6,07 & 16,8 \\
\hline $\begin{array}{l}\text { Consommation d'aliment } \\
\text { d'allaitement de } 8 \text { jours }\end{array}$ & & & & & & & \\
\hline à l'abattage $\ldots \ldots \ldots \ldots$ & 0,672 & 0,715 & 0,002 & 0,085 & 97,0 & 6,00 & 16,5 \\
\hline Indice de consommation & $-0,018$ & 0,388 & 0,053 & 0,776 & 75,6 & 1,24 & 8,3 \\
\hline Taille de la portée.... & $-0,105$ & $-0,049$ & $-0,453$ & $-0,315$ & 31,8 & 2,16 & 23,8 \\
\hline Poids de la carcasse ..... & 0,926 & 0,210 & 0,245 & $-0,041$ & 96,3 & 6,70 & 14,0 \\
\hline Rendement de la carcasse & 0,852 & $-0,158$ & $-0,248$ & $-0,034$ & 81,3 & 69,6 & 3,1 \\
\hline $\begin{array}{c}\text { Note de coloration de } \\
\text { viande } \ldots \ldots \ldots \ldots \ldots \ldots \\
\text { Note d'engraissement de }\end{array}$ & 0,313 & $-0,327$ & $-0,031$ & $-0,197$ & 24,5 & 3,69 & 24,2 \\
\hline $\begin{array}{l}\text { Note d'engraissement de } \\
\text { la carcasse } \ldots . \ldots \ldots \ldots\end{array}$ & 0,514 & $-0,183$ & $-0,545$ & 0,285 & 67,6 & 3,69 & 30,3 \\
\hline Note de musculature .... & 0,667 & $-0,109$ & $-0,190$ & $-0,357$ & 62,0 & 3,66 & 24,6 \\
\hline Viscères vides $\ldots \ldots \ldots$ & $-0,677$ & 0,438 & $-0,038$ & $-0,115$ & 66,5 & 7,67 & 9,4 \\
\hline Rumen vide .......... & $-0,612$ & 0,241 & 0,300 & $-0,225$ & 57,3 & 0,81 & 21,6 \\
\hline Caillette vide ......... & $-0,526$ & 0,121 & $-0,092$ & $-0,369$ & 43,6 & 0,89 & 12,2 \\
\hline Peau...$\ldots \ldots \ldots \ldots$ & $-0,538$ & 0,248 & 0,062 & 0,492 & 59,7 & 8,07 & 6,0 \\
\hline Toilette $\ldots \ldots \ldots \ldots \ldots$ & 0,516 & 0,230 & $-0,536$ & 0,309 & 70,2 & 1,48 & 30,4 \\
\hline $\begin{array}{l}\text { Part de variance expliquée } \\
(\%) \ldots \ldots \ldots \ldots \ldots \ldots \ldots \\
\end{array}$ & 39,74 & 13,10 & 10,89 & 8,28 & & & \\
\hline $\begin{array}{l}\text { Part de variance expliquée } \\
\text { cumulée }(\%) \quad \ldots \ldots \ldots \ldots\end{array}$ & 39,74 & 52,84 & 63,74 & 72,02 & & & \\
\hline
\end{tabular}

Les nombres en italique indiquent les 2 corrélations les plus élevées entre les caractères et les composantes. 
ne sont donc que moyennement liées à celles des poids, des vitesses de croissance, de l'indice de consommation et du rendement de la carcasse. L'étude des sorties graphiques permet de préciser la nature de ces liaisons.

Sortie graphique des composantes 1 et 3 (fig. 2).

La première composante représente un facteur de variation prépondérant puisqu'il explique 39,7 p. Ioo de la variance des données. D'après les positions des caractères bien expliqués de poids, de croissance, de rendement et d'appréciation de la carcasse opposées à celles des constituants du cinquième quartier, il apparaît sur ce plan, qu'indépendamment de l'âge à l'abattage et de l'indice de consommation placés près du centre, les animaux les plus lourds à l'abattage sont évidemment ceux qui ont eu une croissance rapide et qui présentent une musculature et un engraissement les plus satisfaisants; ce qui confirme les corrélations élevées entre ces caractères rapportés au tableau 5 . Ces animaux ont, en outre, un meilleur rendement de carcasses car la proportion relative des principaux constituants de leur cinquième quartier: viscères totaux, rumen, caillette et peau, est faible.

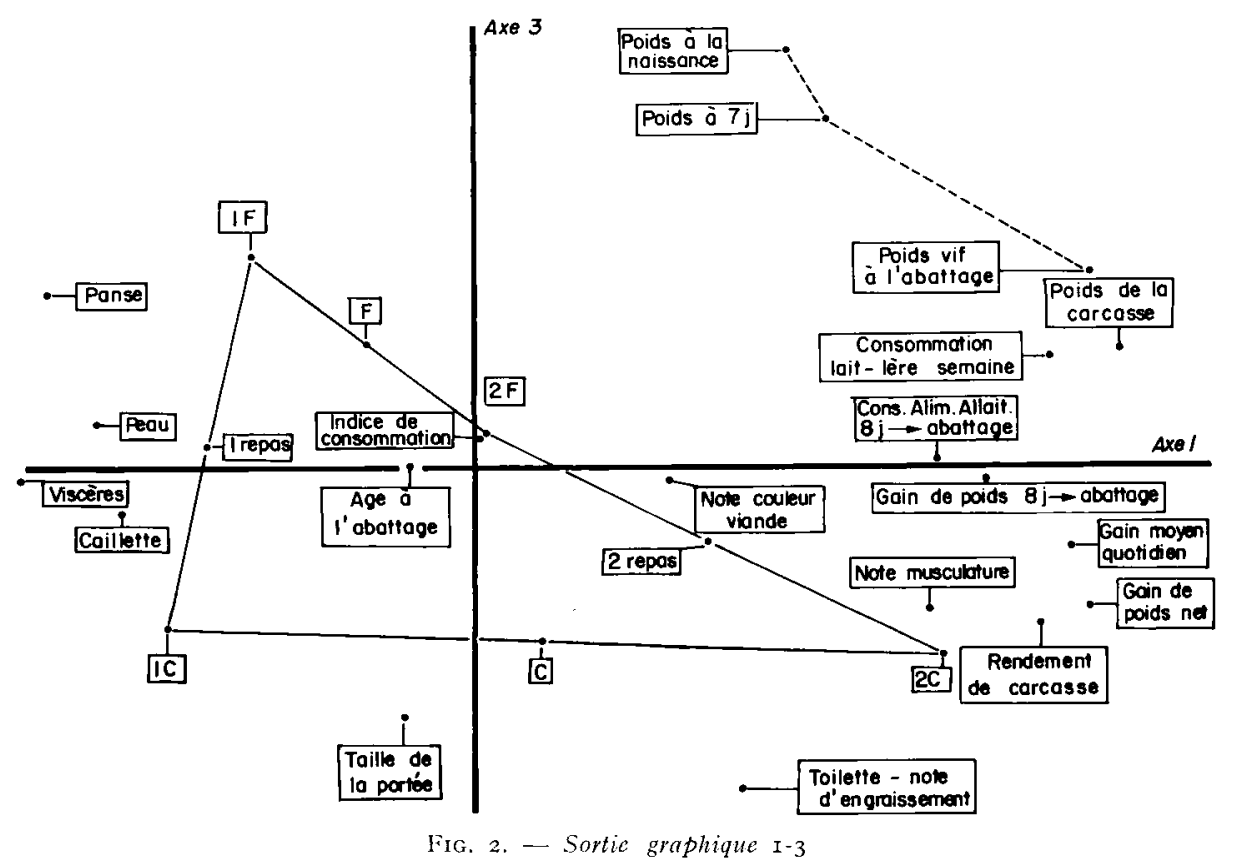

Cette composante révèle aussi que les animaux les plus lourds à la naissance, indépendamment de la taille de la portée, ont tendance à croître plus rapidement ; ce qui confirme la corrélation positive existant entre le poids à la naissance et le poids à l'abattage (tabl. 5).

Une analyse de variance effectuée à partir des abscisses des observations, c'està-dire, à partir de celles des 32 chevreaux regroupés suivant le plan expérimental sur le premier axe permet de conclure que le nombre de repas est un des facteurs discriminants de cet axe (tabl. 7). Bien que l'interaction entre le nombre de repas et la 
température du lait ne soit pas significative, la disposition des centres de gravité de chaque lot sur la première composante montre que les animaux du lot $2 \mathrm{C}$ ont des performances très sensiblement supérieures à celui des autres lots. La signification de la troisième composante est principalement liée aux différences de poids à la naissance dues à la taille de la portée. La disposition des poids et des gains de poids sur l'axe 3 indique que l'effet de la taille de la portée sur le poids des chevreaux s'estompe, en partie, pendant la phase de croissance étudiée. La consommation des chevreaux les plus lourds à la naissance, en effet, n'est pas plus élevée pendant la période expérimentale. Par ailleurs les animaux nés légers en raison de la dimension de la portée à laquelle ils appartiennent, présentent un meilleur état d'engraissement. Cependant, la tendance des animaux à se répartir sur cet axe de façon légèrement inégale suivant la température du lait (tab1. 7) ne permet pas de généraliser systématiquement ces observations à toute population de chevreaux.

\section{TABI.EAU 7}

Analyses de variance effectuées à partir des abscisses des points-observations (chevreaux) sur les quatre premières composantes principales

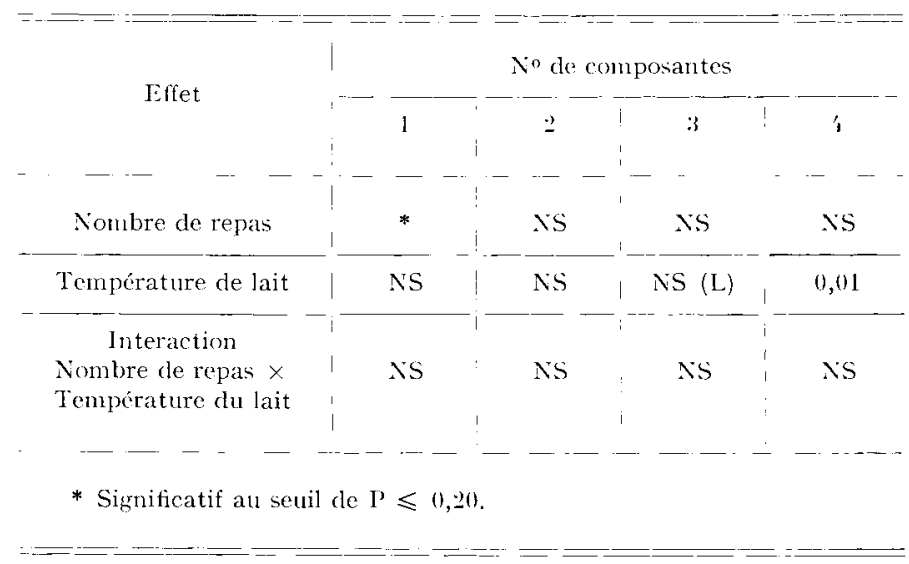

Sortie graphique des composantes 2 et 4 (fig. 3).

La seconde composante est due aux différences d'âge à l'abattage des animaux, indépendamment de la taille de la portée et du poids à la naissance. Les chevreaux abattus tardivement sont plus lourds, ils ont un indice de consommation élevé, une viande plus colorée et un cinquième quartier plus important. De plus, ils semblent avoir démarré assez lentement. Par ailleurs, de bonnes performances des cherreaux durant la première semaine se répercutent favorablement à l'abattage sur les qualités bouchères de leurs carcasses. La proportion de toilette et 1'évaluation subjective de l'engraissement ne sont discriminées que sur cette deuxième composante. Tout se passe comme si un médiocre démarrage suivi d'une croissance compensatrice partielle ne favoriserait pas l'état d'engraissement général de l'animal mais seulement le développement de certains tissus adipeux, tel que la toilette.

Bien que les animaux du lot $2 \mathrm{C}$ aient été abattus tardivement (tab1. 3) et malgré 
leur position dans ce plan, l'analyse de variance réalisée à partir des projections des observations sur la deuxième composante indique que le facteur de variation représenté par cette composante est indépendant des deux paramètres expérimentaux : nombre de repas et température du lait (tabl. 7).

I, a quatrième composante souligne l'opposition entre les effets les plus caractéristiques du lait froid et du lait chaud (tabl. 7). Elle met en particulier en évidence que l'ingestion de lait froid abaisse l'indice de consommation, le poids de la peau et'l'état d'engraissement des animaux.

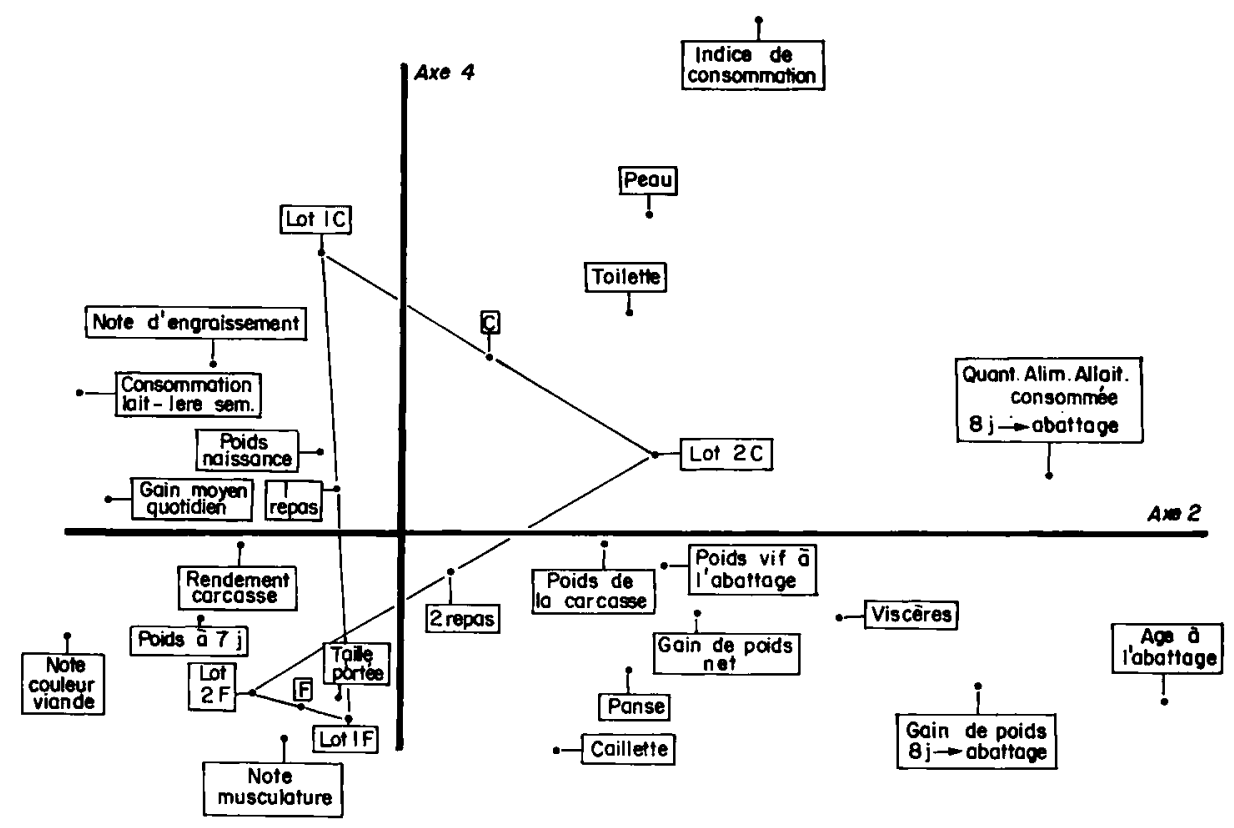

FIG. 3. - Sortie graphique 2-4

\section{DISCUSSION E'T CONCLUSION}

L'analyse en composantes principales a permis de distinguer les interrelations entre les caractères étudiés et de prendre réellement en compte l'effet de facteurs de variation autres que le nombre de repas et la température du lait, notamment, la taille de la portée, l'âge à l'abattage, le poids de naissance et la phase de démarrage de la croissance. Elle a pu ainsi mettre en évidence les caractéristiques de la croissance du chevreau mâle pendant le premier mois en dissociant en particulier les composantes de la variation de différents caractères.

Cette analyse a précisé que le poids à la naissance peut varier non setlement en fonction de la taille de la portée, mais aussi suivant des facteurs vraisemblablement d'origine génétique. Dans le premier cas, les différences s'estompent et dans le second, au contraire, elles s'accentuent à mesure que les chevreaux prennent du poids. En effet, d'après la figure 2 , les animaux issus d'une portée importante et, de ce fait, 
plus légers à la naissance, compensent leur faible poids au démarrage par une vitesse de croissance élevée qui a probablement pour conséquence un état d'engraissement supérieur à l'abattage.

Par ailleurs, l'opposition entre le poids relatif des viscères d'une part, et le niveau de consommation, les gains de poids et le rendement de la carcasse d'autre part (fig. 2) suggèrent que le poids absolu des viscères est relativement indépendant des quantités de lait ingérées et de la vitesse de croissance.

En outre, la valeur des jugements subjectifs de certains critères de qualité de la carcasse a pu être testée grâce à l'analyse utilisée. En particulier, la note de musculature est étroitement liée au rendement de la carcasse et au gain de poids moyen quotidien sur les trois premières composantes. La note d'engraissement se projette au même point que le poids de toilette sur les axes I, 3 et 4 . Toutefois, la seconde composante montre que ces deux variables ne donnent pas toujours la même information sur l'engraissement des animaux. En effet, la note d'engraissement caractérise mieux les animaux dont la croissance a bien démarré tandis que la proportion de toilette est plus importante chez les animaux ayant ingéré assez peu de lait pendant la première semaine et qui ont été abattus tardivement.

D'après nos observations journalières, la distribution d'un repas au lieu de deux repas par jour ne provoque ni une augmentation des cas de diarrhées ni une diminution de la vigueur des chevreaux comme ACKERman et al. (I969), BuRT (I968), OWENS et STAKe (I97 I), OWFN et al. (I965), WilizerT et al. (I969), WrLSON (I968), White et RADClifFFE (I970), à l'exception de WoODEN et al. (I968) et de RADMALL et AdAms (I97I), l'ont déjà observé chez le veau. Or, chez cet animal, on constate parfois une augmentation des cas de diarrhées et une diminution de 1'utilisation de la matière sèche quand la concentration du lait de remplacement atteint 25 p. IOO (PETTYJOHN et al. I963) ou qu'il ingère de trop grandes quantités de lait par repas (Roy, I964) ; ce n'est pas le cas chez le chevreau lorsqu'il lui est distribué à volonté un seul repas par jour de lait à $23 \mathrm{p}$. Ioo de matière sèche. Une capacité limitée de la caillette ou une trop grande dilution des enzymes digestives dans le volume de lait ingéré serait peut-être moins à redouter chez le chevreau. Ėn définitive, cet animal paraît s'adapter très bien à ne recevoir qu'un repas par jour puisqu'il diminue sa consommation alimentaire et, par conséquent, sa croissance de 5 à 6 p. Ioo seulement. Celle-ci semble dépendre essentiellement, comme chez le veau, (LEAVER et YARROW, I972 $b$ ) de la consommation de matière sèche, quel que soit le nombre de repas distribués. L'efficacité de l'utilisation alimentaire ne paraît pas modifiée, mais le rendement vrai et l'engraissement de la carcasse sont sensiblement réduits ; la "finition " de l'animal est donc légèrement moins satisfaisante.

La basse température du lait de remplacement en limitant la consommation alimentaire du chevreau ralentit sa croissance pendant la période d'adaptation dont la durée est modulée par le nombre de repas distribué. Les résultats obtenus sur agneaux ou sur veaux varient sensiblement selon le mode de distribution : restreint ou ad libitum, en repas ou à la disposition de l'animal pendant toute la journée. PENNING et al. (I972) et TAYLER et LONDSALE (I969) enregistrent une baisse de la consommation alors que GibB et PenNing (1972) observent une augmentation de la consommation lorsque la température du lait diminue. De ce fait, la croissance peut être inférieure (TAYLER et LONDSALE, I969; FliPOT et al., I972, égale (BRISSON et BOUChard, I970; GrbB et PENNING, I972) ou supérieure (RADMALI et AdAMS, I97I ; 
LARGE et PENNIng, I967) à celle enregistrée avec du lait chaud selon d'une part, l'aptitude des animaux à consommer du lait froid qui, d'après nos résultats, peut évoluer favorablement dans le temps et selon, d'autre part, la variation de l'efficacité de l'utilisation alimentaire. Alors que l'énergie nécessaire à réchauffer les ingestats devrait plutôt abaisser l'efficacité de 1'utilisation alimentaire, la distribution de lait froid semble l'améliorer chez le chevreau comme parfois chez l'agneau (LARGE et Penning, 1967). Toutefois, Holmes (I97I) en mesurant la consommation d'oxygène observe que l'effet du lait froid sur le bilan énergétique est moindre que celui qui pouvait être prévu. D'autre part, dans nos conditions expérimentales, la présence d'un chauffage par infra-rouge au moment des repas dans les cases des chevreaux recevant du lait froid ne serait pas étrangère à cette amélioration de l'efficacité alimentaire.

L'ingestion de lait froid limite l'engraissement des animaux de façon comparable à la distribution d'un seul repas par jour en raison de leur plus faible consommation. Enfin, tout comme Brisson et Bouchard (I970) et à la différence de RADMALI et Admaxs (I97I) aucune diarrhée n'a été observée sur les animaux recevant du lait froid.

Les effets du nombre de repas et de la température du lait s'ajoutent en général puisque leur interaction est très rarement significative. En particulier, la consommation, le gain de poids et l'engraissement du lot I F sont encore plus faibles que ceux des lots $\mathrm{I} C$ et $2 \mathrm{~F}$. En revanche, son rendement vrai de carcasse est légèrement supérieur à celui des lots I $\mathrm{C}$ et $2 \mathrm{~F}$.

En dépit du gain de poids journalier, plus faible de 5 p. Ioo environ lorsque du lait froid est distribué au chevreau, et aussi du moindre engraissement des carcasses, il semble bien que l'une ou l'autre de ces méthodes puisse être intéressantes à utiliser dans la production de chevreau de boucherie. En effet, la qualité des carcasses reste très acceptable, notamment la couleur de la viande. D'autre part, l'effet légèrement défavorable sur la croissance peut être éliminé par un seul jour supplémentaire d'engraissement qui permet d'atteindre le même gain de poids global que celui des chevreaux recevant 2 repas de lait chand. Par ailleurs, la distribution de lait froid dans les conditions décrites, améliore l'efficacité alimentaire et, de ce fait, le coût de l'alimentation, alors que le coût supplémentaire par chevreau dî au chauffage pendant les repas reste faible. En outre, bien que les conditions expérimentales aient empêché d'effectuer des mesures précises, on peut estimer que la distribution d'un seul repas par jour et de deux repas de lait froid à des chevreaux fait gagner respectivement plus de 30 et 20 p. Ioo de la main-d'œuvre employée à la distribution des repas.

En revanche, la méthode qui consiste à distribuer un seul repas de lait froid semble provoquer un ralentissement trop prolongé de la croissance et donner des carcasses " insuffisamment couverte ". Cette méthode peut plus difficilement être employée en production de chevreaux de boucherie, excepté si la disponibilité de main-d'œuvre est vraiment le principal facteur limitant de l'exploitation. 


\section{SUMMARY}

\section{SEPARATE AND CUMULATED EFFECTS OF THE NUMBER OF MEALS AND OF THE MILK TEMPERATURE \\ ON THE MEAT PRODUCTION PERFORMANCES OF KIDS}

Thirty-two male kids of the Alpine breed were divided into + groups receiving from day 9 a milk replacer solely, according to the following schedule :

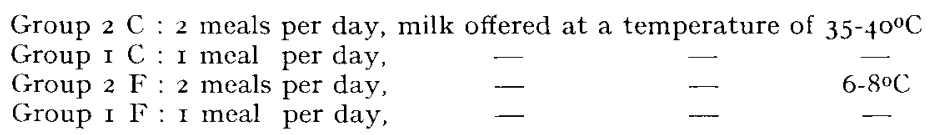

The diet of groups $2 \mathrm{C}$ and $2 \mathrm{~F}$ contained $\mathrm{I} 6 \mathrm{p}$. I oo milk substitute, that of groups $\mathrm{IC}$ and $\mathrm{r}$, $24 \mathrm{p}$. Ioo. The animals fed with cold milk had the possibility of going under an infra-red lamp.

The kids were slaughtered at a weight ranging between 9 and $10.5 \mathrm{~kg}$ and at an age of 28 to 37 days.

Daily feeding of one meal per day instead of two and consumption of cold milk slightly reduced the daily feed intake, the growth rate and fatness of the carcasses. The ingestion of cold milk improved the feed efficiency. The effect of the number of meals and that of the temperature of the milk were cumulated in most cases.

Feeding of one only meal per day or of cold milk seem to be valuable techniques to be used in the production of kids for slaughter because of the economy of labor and satisfactory level of performances obtained.

Besides, the data interpretation on the basis of principal components analysis resulted in a better determination of grow th characteristics in male kids during the first month of life.

\section{RÉFÉRENCES BIBLIOGRAPHIQUES}

Ackerman R. A., Thomas R. O., Thayne W. V., Butcher D. F., ig69. Effects of once a day feeding of milk replacer on body weight of dairy calves. J. Dairy, Sci., 52, I869-I872.

Bettenay R. A., I97I. Dairy calves can safety be fed once a day. J. Agric. (Western Australia), 12, 33-35.

Brisson G. J., Bouchard R., i970. Artificial rearing of lambs: feeding cold milk " ad libitum versus » warm milk three times par day to appetite, and effects of an antibiotic-vitamin-iron supplement on growth performance and digestibility of diet constituents. J. Anim. Sci, 31, 810-815.

Burt A.W. A., r968. A note on the effect of giving milk substitute only once a day to early-rveaned calves. Anim. Prod., 10, I 13 -1 6 6.

Disset R., I97I. Types de chevreaux à produire et leur prix de revient. Journée d'étude sur l'alimentation des jeunes caprins, 22 avril 1971 , Paris, I. N. A., 86-92.

Eecknout W., Buysse $F_{\text {. }}$ 1969. L'influence de l'approvisionnement en lait artificiel en un ou deux repas par jour lors de l'élevage de veaux mâles destinés à la production intensive de viande. Rev. A gric. (Bruxelles) 22, I I I-I I 23.

Eeckhout $\mathbb{W}^{\circ}$., Buysse $\mathrm{F}$., I97r. L'influence de la distribution de lait reconstitué en un ou deux repas par jour à des veaux mâles destinés à la production intensive de viande. Rev. A gric. (Bruxelles), 24, I 56 I-I 570 .

FEIik P. M., I97I. Influence du nombre de repas et de la température du lait de remplacement sur les performances des chevreaux de boucherie. Xe Cong. Intern. Zoot., Versailles, 20-23 juillet 1971. Thème VII. Nutrition ruminants.

Fltpot P., Lalande G., liarmy M. H., r972. Fffects of temperature of milk replacer and method of feeding on the performance of Holstein veal calves. Can. J. Anim. Sci., 52, 659-664.

Gibb M. J., Pexxing P. D., I972. The effects of environmental temperature and feeding pattern on the artificially reared lambs fed cold milk substitute. Anim. Prod., 15, 177-182.

Hodex A., Jourset M., I972. Distribution à des veaux d'élevare de lait reconstitué en une ou deux fois par jour de la naissance au sevrage. Bull. Techn. C. R. Z. I., Theix, mars 1972 (7) 23-30.

Holmes C. W., I97I. The effect of milk given at various temperature on the oxygen consumption of young calves. Anim. Prod., 13,619-625. 
Khouri R. H., I969. The feeding of milk once daily to early weaned calves. N. Z.J. Agric. Res., 12, $650-654$.

LaRge R. V., Penning P. D., 1967. The artificial rearing of lambs on cold reconstitued whole milk and on milk substitute. J.Agric. Sci. (Camb.), 69, 405-409.

Leaver J. D., Yarrow N. H., I972 a. Rearing of dairy cattle. I. Type and level of milk substitute offered once daily to calves. Anim. Prod., 14, I55-159.

Leaver J. D., Yarrow N. H., I972 b. Rearing of dairy cattle. III. A note on the use of whole milk for calves fed once daily. Anim. Prod., 15, 315-3 8 .

Owen F., Plum M., Harris L., I965. Once versus twice daily feeding of milk to calves at $2 \mathrm{I}$ or 42 days of age. J. Dairy Sci., 48, 824 (Abst.).

Owens M. J., Stake P. E., I97r. Once versus twice daily milk feeding of dairy calves. J. Dairy Sci., 54, 8 or (Abst.).

Penning P. D., Bradfield P. G. E., Treacher T. T., I97I. A note of the performance of artificially reared lambs fed cold milk substitute from birth to slaughter. Anim. Prod., 13, 365-368.

Penning I. M., Treacher T. T., Penning P. D., ig7z. The effect of feeding regimes and milk substitute temperature on the performance of artificially reared lambs. Proc. Br. Soc. Anim. Prod., I972, I 44 (Abst.).

Pettyjohn J. D., Everett J. P. Mochrie R. D., i963. Responses of dairy calves to milk replacer fed at various concentrations. J. Dairy Sci., 46, 7I0-714.

Radmall M. B., Adams H. P., I97I. Feeding frequency, milk temperature and calf growth. J. Anim. Sci., 33, 297 (Abst.).

Roy J. H. B., I964. The nutrition of intensively reared calves. Vet. Res., 76, 5 II-526.

Sauvant D., Fehr P. M., Rodolphe F., Tomassone R., Delage J., I973. Étude des interrelations entre les critères de production et de composition lipidique du lait de chèvre par deux méthodes d'analyse factorielle. Ann. Biol. anim. Bioch. Biophys., 13, 107-129.

Tayler J. C., Londsale C. R., I969. The artificial rearing of calves and their growth on grass diets. I. The effect of the type and temperature of milk substitute given ad libitum. J. A gric. Sci., 73, 279-287.

Theriez M., Molenat G., I969. L'allaitement artificiel de l'agneau. Session d'études A.F.Z., 16-18 décembre 1971. Tome II, rapport 38 .

White B. R., Radcliffe J. C., r970. A comparison of once daily and twice daily feeding of milk replacers to dairy calves. Proc. Austr. Soc. Anim. Prod., 8, 247-25I.

Willett L. B., Albright J. L., Cunningham M. D., ig69. One-versus twice-daily feeding of milk replacer to calves. J. Dairy Sci, 52, 390-39r.

Wilson J., I968. Once daily feeding of calves. Vet. Res., 83, $42-43$.

WoOd A. S., BAyley H. S., MACLEOD G. K., r97r. Evaluation of imposing a weekly fast on calves receiving a milk replacer diet once and twice daily: Protein and energy utilization. J. Dairy Sci, 54, 405-4II.

Wooden K. G., Speicher J. A., Huber J. T., I968. Effect of feeding systems on feed and labor costs and rates of grain in dairy calves. J. Dairy Sci, $\mathbf{5 1}, 97 \mathrm{I}$ (Abst.). 